\title{
Somatostatin and diabetic retinopathy: an evolving story
}

\author{
Olga Simó-Servat ${ }^{1,2} \cdot$ Cristina Hernández ${ }^{1,2} \cdot$ Rafael Simó ${ }^{1,2}$
}

Received: 31 January 2018 / Accepted: 4 February 2018 / Published online: 20 February 2018

(c) Springer Science+Business Media, LLC, part of Springer Nature 2018

The story of how somatostatin (SST) has come to play an evolving role in the treatment of diabetic retinopathy (DR) has to be seen in the context of the growth of scientific knowledge regarding the pathophysiology of DR.

The clinical observation published by Poulsen in 1953 [1] reporting that a patient with Sheehan's syndrome presented a partial regression of proliferative diabetic retinopathy (PDR), and its post-mortem confirmation 13 years later [2] were essential preconditions for the theory that hypohysectomy could be a treatment for PDR. The studies published in 1970 by Merimee et al. [3] showing that GH (growth hormone) administration in diabetic patients with GH deficiency stimulated the development of DR led to the plausible mechanistic hypothesis that the effectiveness of hypophysectomy was mediated by decreased circulating levels of GH/insulin-like growth factor(IGF)-1. In fact, yttrium-90 pituitary implantation [4] and hypophysectomy [5] were proposed as treatments for PDR. However, it was unclear whether the observed benefit might have been related to improved blood glucose control after hypophysectomy rather than to the GH/IGF reduction itself. In addition, the results reported by Merimee et al. [3] could have been due to the worsening of metabolic control after GH administration. Furthermore, with the advances of treatment for PDR, such as laser photocoagulation, this type of aggressive approach became obsolete.

The extensive investigation and clinical use of somatostatin analogues (SSA) over the past 30 years has provided a good opportunity to test again in a more appropriate and safer manner of the hypothesis that the inhibition of

Rafael Simó

rafael.simo@vhir.org

1 Diabetes and Metabolism Research Unit, Vall d'Hebron Research Institute, Barcelona, Spain

2 Centro de Investigación Biomédica en Red de Diabetes y Enfermedades Metabólicas Asociadas (CIBERDEM), Instituto de Salud Carlos III (ISCIII), Barcelona, Spain
GH/IGF-1 could arrest the progression of DR. In the beginning of this century, pilot studies using SSA administered intramuscularly in patients with early PDR and severe non-proliferative DR (NPDR) revealed a decreased incidence of progression to PDR requiring panretinal laser treatment [6] or vitreo-retinal surgery [7]. However, a randomized double-blind placebo-controlled study with two arms (study 1 [NCT00131144] conducted in Europe using long-acting octreotide 20 and $30 \mathrm{mg}$, and another in North America and South America [NCT00130845] using longacting octreotide $30 \mathrm{mg}$ ) with an overall randomization of 899 patients completed in 2006 did not confirm these results [8]. This study revealed that long-acting octreotide given intramuscularly every 4 weeks in moderate-to-severe NPDR to low-risk PDR patients was unable to arrest DR progression significantly. The cohorts included in this clinical trial were recently used by Pivonello et al. [9] to evaluate the long-term safety of long-acting octreotide by means of a targeted post-hoc analysis. The authors confirmed the established cardiac, hepatic, and renal safety profile of longacting octreotide in diabetic patients with DR with a median duration of treatment of over 3.5 years. This is an important finding because DR is an independent predictor of diabetic nephropathy, cardiovascular disease, and stroke [10, 11], thus increasing even the risk of morbi-mortality of T2D subjects. Therefore, the lesson drawn from this paper regarding the safety of long-term administration of an SSA in a population with a high risk of suffering co-morbidities, is very relevant for the clinical practice.

However, the reasons why long-acting octreotide failed to arrest the progression of DR remain to be elucidated. There are several important points which deserve to be commented on, and that could help us to shed light on this issue:

1) The role of circulating IGF-1 in the development and progression of DR is controversial, and this concept has not been supported by either large cross-sectional [12] or prospective clinical trials $[13,14]$. In fact, acromegalic patients with diabetes do not present an increased incidence of DR, and when it appears it is not more aggressive [15]. 
Therefore, the inhibition of GH/IGF-1 circulating levels does not seem to be a good target for preventing or arresting DR.

2) Rather than systemic levels, the local production of IGF-1 in the diabetic retina could be involved in the pathogenesis of DR. Although there is extensive experimental evidence that appear to support this idea, the studies have been performed using supraphysiological concentrations of IGF-1 and/or using non-diabetic experimental models [16]. In addition, mRNA levels have been found lower in the retinas of diabetic donors in comparison with age-matched non-diabetic donors [17]. Therefore, the use of SSAs based on their potential effect in reducing the intraocular production of IGF-1 also does not seem to be a reasonable approach to treating DR.

3) SST has neuroprotective and antiangiogenic effects unrelated to IGF-1 [16]. In addition, SST participates in the regulation of water and ions transport [18]. Therefore, it can be postulated that SST could prevent neurodegeneration, neovascularization, and vascular leakage, all of them are the key players in the development of DR. However, octreotide does not cross the blood-retinal barrier (BRB) and has access to the retina only when there is a breakdown of the $\mathrm{BRB}$, thus limiting the amount of the drug reaching the retinal target tissues.

In recent years it has been demonstrated that the retina, and in particular the retinal pigment epithelium, is a significant source of SST [19]. In fact, SST levels within the vitreous fluid are higher than in plasma [20]. In the retina from diabetic patients there is a downregulation of SST and it is related to glial activation and neuron apoptosis, the two hallmarks of retinal neurodegeneration [19]. Thus, SST replacement treatment can be envisaged as a reasonable approach for treating DR. Since SSAs may have inadequate penetration of the BRB, local delivery of SST or SST agonists might be a potential route of administration. However, when the early stages of DR are the therapeutic target, it would be inconceivable to recommend aggressive treatments such as intravitreal injections. The use of topical administration (i.e., eye drops) has traditionally been considered inappropriate for treating diseases of the posterior chamber of the eye, such as in the case of DR. However, there is emerging experimental evidence that many drugs are able to reach the retina and exert pharmacological effects in terms of preventing neurodegeneration, inflammation, and/or vascular leakage after their topical administration [21-23].

SST was one of the first reported drugs with experimental neuroprotective action after its topical administration [21]. This preclinical evidence together, with the successful approval of the current regulatory requirements, permitted the first clinical trial aimed at evaluating the effects of topically administered neuroprotective agents (SST and brimonidine) in diabetic patients with no or mild DR to be implemented (NCT01726075). This was a randomized, placebo-controlled, phase II-III trial of parallel groups. A total of 450 patients with type 2 diabetes were enrolled at 11 European centers belonging to the EUROCONDOR consortium [24]. Eligible patients $(n=449)$ were randomly allocated in a 1:1:1 ratio to one of 3 arms: Group A: placebo (1 drop BID in each eye), Group B: somatostatin $0.1 \%$ (1 drop BID in each eye), and Group C: brimonidine tartrate $0.2 \%$ ( 1 drop BID in each eye). The primary outcome was the change in Implicit Time (IT) assessed by multifocal electroretinography (mfERG) between baseline and at the end of follow-up (96 weeks). This clinical trial revealed that SST and brimonidine were only useful in the subset of patients with pre-existing retinal neurodysfunction, in which the worsening of IT was prevented [25]. This finding points to screening retinal neurodysfunction as a critical issue to identify a subset of patients in whom neuroprotective treatment might be of benefit. It is worth mentioning that neuronal integrity is essential for vision, and in the early stages of diabetes many patients present deficiencies such as decreased hue discrimination and contrast sensitivity, delayed dark adaptation, abnormal visual fields, and impairment of vision related quality of life with specific reference to colour and peripheral vision [26]. Therefore, neuroprotection itself can be considered a therapeutic target, independently of its potential to prevent the development or progression of retinal microvascular disease. The results of the EUROCONDOR clincial trial should be confirmed in further studies using emerging technologies. In addition, a longer follow-up aimed at assessing whether the neuroprotection results in a reduction of retinal microvascular disease is needed.

In summary, the change in the paradigm regarding the use and route of the administration of SST for the treatment of DR provides a good example of our increasing understanding of the pathophysiology of DR. In this regard, we can guarantee that the story will be continued.

Acknowledgements O.S. is a recipient of a "Río Hortega" fellowship grant from the Instituto de Salud Carlos III.

\section{Compliance with ethical standards}

Conflict of interest The authors declare that they have no conflict of interest.

\section{References}

1. J.D. Poulsen, Diabetes and anterior pituitary deficiency. Diabetes 2, 7-12 (1953)

2. J.D. Poulsen, Diabetes and anterior pituitary deficiency: final course and postmortem study of a diabetic patient with Sheehan's syndrome. Diabetes 15, 73-77 (1966) 
3. T.J. Merimee, S.E. Fineberg, V.A. McKusik, J. Hall, Diabetes mellitus and sexual ateliotic dwarfism: comparative study. J. Clin. Invest. 49(6), 1096-1102 (1970)

4. P.S. Sharp, T.J. Fallon, O.J. Brazier, L. Sandler, G.F. Joplin, E.M. Kohner, Long term follow-up of patients who underwent ytrum90 pituitary implantation for treatment of proliferative diabetic retinopathy. Diabetologia 30(4), 199-207 (1987)

5. E.M. Kohner, G.F. Joplin, R.K. Black, H. Cheng, T.R. Fraser, Pituitary ablation in the treatment of diabetic retinopathy. Trans. Ophthalmol. Soc. U.K. 92, 79-90 (1972)

6. M.B. Grant, R.N. Mames, C. Fitzgerald, K.M. Hazariwala, R. Cooper-DeHoff, S. Caballero, K.S. Estes, The efficacy of octreotide in the therapy of severe nonproliferative and early proliferative diabetic retinopathy: a randomized controlled study. Diabetes Care 23(4), 504-509 (2000)

7. B.O. Boehm, G.K. Lang, P.M. Jehle, B. Feldman, G.E. Lang, Octreotide reduces vitreous hemorrhage and loss of visual acuity risk in patients with high-risk proliferative diabetic retinopathy. Horm. Metab. Res. 33(5), 300-306 (2001)

8. S.S. Palii, S. Caballero, G. Shapiro, M.B. Grant, Medical treatment of diabetic retinopathy with somatostatin analogues. Expert Opin. Investig. Drugs 16(1), 73-82 (2007)

9. R. Pivonello, G. Muscogiuri, G. Holder, M. Paul, S. Sarp, A. Lesogor, P. Jordaan, J. Eisinger, A. Colao, Long-term safety of long-acting octreotide in patients with diabetic retinopathy: results of pooled data from 2 randomized, double-blind, placebocontrolled phase 3 studies. Endocrine. https://doi.org/10.1007/ s12020-017-1448-5. (2017)

10. M. Takagi, T. Babazono, Y. Uchigata, Differences in risk factors for the onset of albuminuria and decrease in glomerular filtration in people with Type 2 diabetes mellitus: implications for the pathogenesis if diabetic kidney disease. Diabet. Med. 32(10), 1354-1360 (2015)

11. J.R. Brownrigg, C.O. Hughes, D. Burleigh, A. Karthikesalingam, B.O. Patterson, P.J. Holt, M.M. Thompson, S. de Lusignan, K.K. Ray, R.J. Hinchliffe, Microvascular disease and risk of cardiovascular events among individuals with type 2 diabetes: a population-level cohort study. Lancet Diabetes Endocrinol. 4(7), 588-597 (2016)

12. J.F. Payne, V. Tangpricha, J. Cleveland, M.L. Lynn, R. Ray, S.K. Srivastava, Serum insulin-like growth factor-I in diabetic retinopathy. Mol. Vis. 17, 2318-1324 (2011)

13. Q. Wang, D. Dills, R. Klein, B. Klein, S. Moss, Does insulin-like growth factor I predict incidence and progression of diabetic retinopathy? Diabetes 44(2), 161-164 (1995)

14. Growth Hormone Antagonist for Proliferative Diabetic Retinopathy Study Group, The effect of a growth hormone receptor antagonist drug on proliferative diabetic retinopathy. Ophthalmology 108(12), 2266-2272 (2001)

15. S. Azzoug, F. Chentli, Diabetic retinopathy in acromegaly. Indian J. Endocr. Metab. 18(3), 407-409 (2014)

16. R. Simó, E. Carrasco, M. García-Ramírez, C. Hernández, Angiogenic and antiangiogenic factors in proliferative diabetic retinopathy. Curr. Diabes. Rev. 2(1), 71-98 (2006)
17. C. Gerhardinger, K.D. McClure, G. Romeo, F. Podesta, M. Lorenzi, IGF-I mRNA and signaling in the diabetic retina. Diabetes 50(1), 175-183 (2001)

18. C. Hernández, O. Simó-Servat, R. Simó, Somatostatin and diabetic retinopathy: current concepts and new therapeutic perspectives. Endocrine 46(2), 209-214 (2014)

19. E. Carrasco, C. Hernández, A. Miralles, P. Huget, J. Farres, R. Simó, Lower somatostatin expression is an early event in diabetic retinopathy and is associated with retinal neurodegeneration. Diabetes Care 30(11), 2902-2908 (2007)

20. C. Hernández, E. Carrasco, R. Casamitjana, R. Deulofeu, J. García-Arumí, R. Simó, Somatostatin molecular variants in the vitreous fluid: a comparative study between diabetic patients with proliferative diabetic retinopathy and non-diabetic control subjects. Diabetes Care 28(8), 1941-1947 (2005)

21. C. Hernández, M. García-Ramírez, L. Corraliza, J. FernándezCarneado, J. Farrera-Sinfreu, B. Ponsati, A. González-Rodríguez, A.M. Valverde, R. Simó, Topical administration of somatostatin prevents retinal neurodegeneration in experimental diabetes. Diabetes 62(7), 2569-2578 (2013)

22. C. Hernández, P. Bogdanov, C. Solà-Adell, J. Sampedro, M. Valeri, X. Genís, O. Simó-Servat, M. García-Ramírez, R. Simó, Topical administration of DPP-IV inhibitors prevents retinal neurodegeneration in experimental diabetes. Diabetologia 60(11), 2285-2298 (2017)

23. C. Hernández, P. Bogdanov, L. Corraliza, M. García-Ramírez, C. Solà-Adell, J.A. Arranz, A.I. Arroba, A.M. Valverde, R. Simó, Topical administration of GLP-1 receptor agonists prevents retinal neurodegeneration in experimental diabetes. Diabetes $\mathbf{6 5}(1)$, 172-187 (2016)

24. A.R. Santos, L. Ribeiro, F. Bandello, R. Lattanzio, C. Egan, U. Frydkjaer-Olsen, J. García-Arumí, J. Gibson, J. Grauslund, S.P. Harding, G.E. Lang, P. Massin, E. Midena, P. Scanlon, S.J. Aldington, S. Simão, C. Schwartz, B. Ponsati, M. Porta, M.A. Costa, C. Hernández, J. Cunha-Vaz, R. Simó, European Consortium for the Early Treatment of Diabetic Retinopathy (EUROCONDOR), Functional and Structural Findings of Neurodegeneration in Early Stages of Diabetic Retinopathy: Crosssectional Analyses of Baseline Data of the EUROCONDOR Project. Diabetes 66(9), 2503-2510 (2017)

25. R. Simó, F. Bandello, C. Egan, J. García-Arumí, J. Gibson, J. Grauslund, S.P. Harding, G.E. Lang, P. Massin, E. Midena, P. Scanlon, B. Ponsati, M. Porta, L. Ribeiro, C. Hernández, J. Cunha-Vaz, The European Consortium for the Early Treatment of Diabetic Retinopathy (EUROCONDOR). Topical administration of somatostatin and brimonidine in the early stages of diabetic retinopathy: results of the EUROCONDOR study. Diabetologia 60(Suppl 1), 1 (2017). https://doi.org/10.1007/s00125-017-4350-z

26. M. Trento, O. Durando, S. Lavecchia, L. Charrier, F. Cavallo, M. A. Costa, C. Hernández, R. Simó, M. Porta, EUROCONDOR trial investigators, Vision related quality of life in patients with type 2 diabetes in the EUROCONDOR trial. Endocrine 57(1), 83-88 (2017) 